\title{
Exploring bitterness of traditional Chinese medicine samples by potentiometric electronic tongue and by capillary electrophoresis and liquid chromatography coupled to UV detection
}

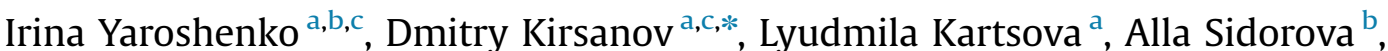 \\ Qiyong Sun ${ }^{\mathrm{d}}$, Haitong Wan ${ }^{\mathrm{e}}$, Yu He ${ }^{\mathrm{e}}$, Ping Wang ${ }^{\mathrm{d}}$, Andrey Legin ${ }^{\mathrm{a}, \mathrm{c}}$ \\ a Institute of Chemistry, St. Petersburg State University, Universitetskaya nab. 7/9, Mendeleev Center, 199034 St. Petersburg, Russia \\ b Bioanalytical Laboratory CSU"Analytical Spectrometry", St. Petersburg State Polytechnical University, Box 27, Gzhatskaya str. 27, 198220 St. Petersburg, \\ Russia \\ ${ }^{\mathrm{c}}$ Laboratory of Artificial Sensory Systems, ITMO University, Kronverkskiy pr., 49, 197101 St. Petersburg, Russia \\ d Zhejiang University, 866 Yuhangtang Road, Hangzhou, Zhejiang Province 310027, China \\ e Zhejiang Chinese Medical University, 548 Binwen Road, Binjiang, Hangzhou, Zhejiang Province 310053, China
}

\section{A R T I C L E I N F O}

Article history:

Received 15 September 2015

Received in revised form 25 January 2016

Accepted 27 January 2016

Available online 28 January 2016

Keywords:

Traditional Chinese Medicine (TCM)

Bitterness

Polyphenols

Chromatographic and electrophoretic pro-

files

Electronic tongue

\begin{abstract}
A B S T R A C T
Instrumental bitterness assessment of traditional Chinese herbal medicine (TCM) preparations was addressed in this study. Three different approaches were evaluated, high-performance liquid chromatography coupled to UV detector (HPLC), capillary electrophoresis coupled to UV detector (CE) and a potentiometric multisensor system - electronic tongue (ET). Most studies involving HPLC and CE separations use these as selective instruments for quantification of individual substances. However we employed these techniques to provide chromatographic or electrophoretic sample profiles. These profiles are somewhat analogous to the profiles produced by the ET. Profiles from all instruments were then related to professional sensory panel evaluations using projections on latent structures (PLS) regression. It was found that all three methods allow for bitterness assessment in TCM samples in terms of human sensory panel with root mean squared errors of prediction ca. 0.9 within bitterness scale from 0 (no bitterness) to 6 (maximal bitterness).
\end{abstract}

(c) 2016 Elsevier B.V. All rights reserved.

\section{Introduction}

Traditional Chinese medicine (TCM) is a very popular approach for therapeutic and preventive treatment of various diseases both within and outside China based on the experience accumulated through thousands of years of practice. An important part of TCM practice is the use of herbal medicines. In the last few decades TCM has attracted considerable attention from pharmaceutical companies as a potential source for discovery of bioactive compounds [1]. This interest required an extensive development of analytical methods for characterization of the constituents of such herbal medicinal products, recent progress in the field being reviewed in [2]. The problem of taste of TCM is closely related to the acceptance of drugs by patients just as in the case of Western pharmaceutical products. TCM preparations are natural products derived from numerous plants and they may have bitter taste [3-

\footnotetext{
* Corresponding author at: Institute of Chemistry, St. Petersburg State University, Universitetskaya nab. 7/9, Mendeleev Center, 199034 St. Petersburg, Russia.

E-mail address: d.kirsanov@gmail.com (D. Kirsanov).
}

6]. One obvious source of bitter taste is the presence of phenolic, polyphenolic substances and alkaloids, which are responsible for bitterness in other plant-derived products like wine [7-9], tea [10$14]$, coffee $[15,16]$, olive oil $[17,18]$, etc. Taste assessment protocols normally include human sensory panels, which are widely known to suffer from sensory fatigue effect, subjectivity and variability due to the individual physical and psychological condition of panelists $[19,20]$. In order to circumvent these issues there is a growing interest in development of instrumental taste assessment methods [21]. The results of various instrumental analytical measurements can be related to sensory panel scores. It was shown that chromatograms [7,22,23], various spectra [24-27] and electropherograms [28] can be employed for prediction of taste attributes in various food samples. These methods are able to predict taste parameters in the analyzed samples due to their high sensitivity towards particular substances responsible for specific taste sensation (e.g. polyphenols [7-14] and caffeine $[15,16,18]$ are known to elicit bitter taste). Another popular approach for instrumental taste assessment is the application of "electronic tongue" systems [29]. These systems are typically based on ensembles of cross-sensitive chemical sensors (potentiometric, voltammetric 
etc.) and their response in the samples is usually treated with chemometric techniques. Taste parameters are predicted from "unresolved" data without direct knowledge about the content of specific taste stimuli in the case of e-tongues. Such systems have already been proved useful for pharmaceutical tasks, e.g. in estimation of taste masking efficacy [30] and assessment of bitter taste of various formulations [31]. Various e-tongues have also been applied for evaluation of TCM samples [4-6]. Classification of various medicinal plants and TCM samples according to their taste was reported in [4]. The e-tongue system comprised five lipid membrane sensors in this work. Taste parameters of an individual substance pretty typical for TCM - berberine were studied by various multisensor systems in $[5,6]$. However, numerical prediction of sensory scores in TCM samples has not yet been studied.

The purpose of the present study was to elaborate the approaches for instrumental taste evaluation of TCM herbs in terms of correlation with a human sensory panel. To fulfill the task we employed three different analytical techniques: capillary electrophoresis coupled to UV detector (CE), high performance liquid chromatography coupled to UV detector (HPLC) and potentiometric multisensor system - the so called "electronic tongue" (ET). Unlike other studies where CE and HPLC were used to provide for concentrations of particular substances we employed these techniques in a way similar to ET - we used the raw chromatographic and electrophoretic profiles derived from UV detection without peak assignment as inputs for projection on latent structures (PLS) modeling and bitterness prediction. The results of TCM drug's taste evaluation by professional sensory panel were taken as referent values for the calibration of these models.

\section{Experimental}

\subsection{Chemicals and materials}

1,8-dihydroxyanthraquinone (95.5\%), epicatechin (EC) (99\%), gallic acid (GA) ( $\geq$ 97.5\%), caffeine (Caf) (99\%), hydrochloric acid $37 \%$, sodium dihydrophosphate dihydrate $(99 \%)$ were purchased from Sigma Aldrich (Germany). Short codes (such as EC, GA, etc.) are used here and below only for substances further mentioned in Tables and Pictures. Epigallocatechin (EGC) ( $\geq 90 \%$ ), epigallocatechin gallate (EGCG) ( $\geq 97 \%$ ), sodium hydrophosphate dodecahydrate (98\%) and formic acid (98\%) were from Fluka (Germany), sodium hydroxide (99\%) and methanol were from Merck, rhein (88.7\%) was from LKT Laboratories (USA), berberine hydrochloride ( $>98 \%$ ) from Northeast Pharmaceutical Group Shenyang NO.1 Pharmaceutical Co., Ltd (China), sodium dodecyl sulfate ( $>98 \%$ ) from Reahim and ethylacetate from Vekton (both St. Petersburg, Russia), acetonitrile from Biosolve (France), TWEEN 20 (>98\%) from Helicon (Moscow, Russia). All other chemicals were of analytical reagent grade and were used without further purification.

Water for preparation of buffer and standard solutions was from Milli-Q Advantage A10 purification system (Millipore, Molsheim, France).

\subsection{Bitterness evaluation of TCM samples}

8 various herbal medicine samples were collected for this study: Chinese Chrysanthemum, Liquorice Root, Cassia Twig, Chinese Ephedra, Bitter Apricot Seeds, Rehmanniae Adhesive, Dandelion Root, Chinese Coptis. All samples were collected in the field and provided by Zhejiang Chinese Medical University. Each sample was provided as a dried plant in amount of $300 \mathrm{~g}$. These herbal products are widely used in TCM for treatment of various diseases. Concentrated brews of the samples were prepared and further mixed with water in various ratios to provide a range of concentrations. Then the tastes of the samples were evaluated by professional tasters to assess their mean bitterness intensity using a scale from 0 (no bitterness) to 6 (maximal bitterness). Solutions with different known concentrations of berberine hydrochloride (a typical bitter substance) were evaluated for training purposes.

Bitterness evaluation of TCM samples consisted of two stages. Firstly, the standard aqueous berberine hydrochloride solutions were prepared and corresponding bitterness scores were set, which is shown in detail in Table $1 \mathrm{~S}$ (Supplementary material). Twenty healthy persons (15 men and 5 women 20-30 years of age) took part in this experiment. The volunteers were asked to keep the standard berberine solutions in the mouth for $20 \mathrm{~s}$ and concentrations and bitterness scores for these solutions were made known. The volunteers rinsed the mouth with water between samples and waited for $10 \mathrm{~min}$ before assessing the next sample. The procedure ensured no perceptible carry over between the samples.

The second stage was bitterness evaluation of TCM by human tasters. $20 \mathrm{ml}$ of each TCM under study were tasted in random order (each sample was kept in the mouth for $20 \mathrm{~s}$ ) and bitterness was estimated according to the established scale. The volunteers then rinsed their mouths with water and waited for $10 \mathrm{~min}$ before tasting the next sample. The average bitterness score was calculated for each sample. The applied procedure allowed for negligible effects due to the assessor fatigue.

Details on the samples, dilution rates and estimated bitterness are presented in the Table 1 .

Table 1

Details on the TCM samples employed in the study.

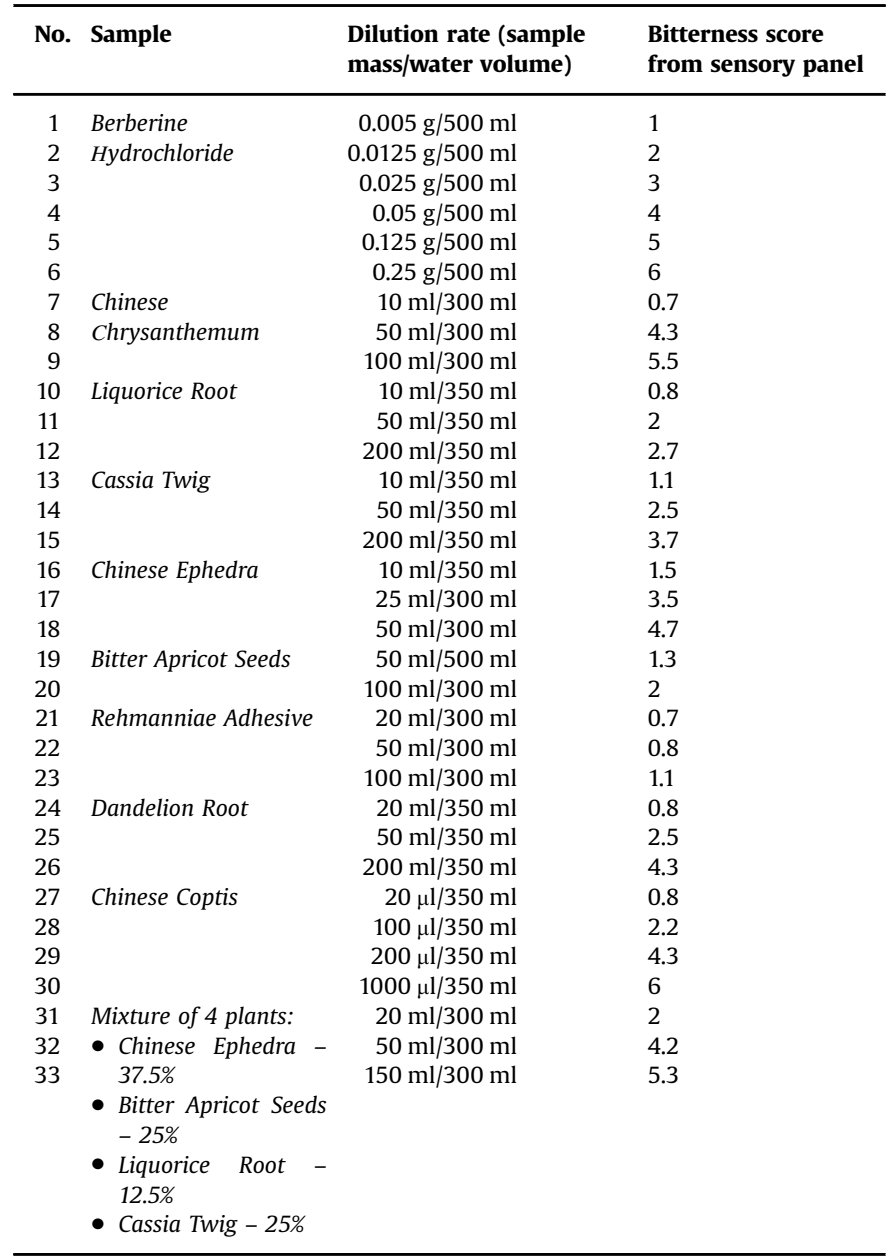




\subsection{HPLC measurements}

HPLC profiling was performed by Agilent-1200 instrument with UV detector (Waldbronn, Germany). Separation was performed using YMC-Triart C18 column $(250 \times 4.6 \mathrm{~mm}, 5 \mu \mathrm{m})$. Aqueous solutions of acetonitrile (60\%) and $0.05 \mathrm{M}$ formic acid were used as mobile phase and the flow rate was set to $0.2 \mathrm{ml} / \mathrm{min}$. The sample injection volume was $40 \mu \mathrm{l}$ and the analysis time was $20 \mathrm{~min}$ at detection wavelength of $254 \mathrm{~nm}$.

Sample preparation for HPLC measurements was performed by vortex mixing of $100 \mathrm{mg}$ of a crushed plant with $1 \mathrm{ml}$ of acetonitrile for 15 minutes. The samples were then centrifuged for $10 \mathrm{~min}$ (Eppendorf 5702R, $4400 \mathrm{rpm}, 3000 \times \mathrm{g}$ ) and $600 \mu \mathrm{l}$ of the upper layer and $400 \mu \mathrm{l}$ of added water were transferred to the glass autosampler vials. These solutions were subjected to chromatographic analysis. Each sample was analyzed three times and averaged HPLC profiles were further taken into account.

\subsection{Capillary electrophoresis instrumentation and conditions}

CE was used for profiling and assessment of total content of polyphenols and caffeine in TCM samples.

CE profiling was performed using a Capel 105 device (Lumex, St. Petersburg, Russia) equipped with UV detector. The separation silica capillary has total and effective lengths of 60 and $54 \mathrm{~cm}$ respectively. The inner capillary diameter was $50 \mu \mathrm{m}$. Standard solutions and samples were diluted with water and were introduced into the capillary hydrodynamically by 30 mbar pressure for $20 \mathrm{~s}$. The experiments were carried out at a constant voltage of $25 \mathrm{kV}$, capillary temperature was $25^{\circ} \mathrm{C}$, detection wavelength was $200 \mathrm{~nm}$ and analysis time was $10 \mathrm{~min}$.

Prior to flushing with running buffer for $15 \mathrm{~min}$ the capillary was preconditioned in the following order: $1 \mathrm{M} \mathrm{HCl}$ for $10 \mathrm{~min}$, deionized water for $10 \mathrm{~min}, 1 \mathrm{M} \mathrm{NaOH}$ for $10 \mathrm{~min}$ and deionized water again for $10 \mathrm{~min}$.

The capillary was washed with running buffer for $5 \mathrm{~min}$ between the samples. This media contained $25 \mathrm{mM}$ phosphate buffer $(\mathrm{pH}=7.0)$ and $25 \mathrm{mM}$ sodium dodecyl sulfate in distilled water.

Sample preparation for CE measurements was performed in the following way: $500 \mathrm{mg}$ of crushed plants dissolved in $10 \mathrm{ml}$ of freshly boiled distilled water were placed into ultrasonic bath ("Sapphire", $1.3 \mathrm{~L}, 35 \mathrm{kHz}$, total power $190 \mathrm{~W}$, heating power $130 \mathrm{~W}$, Moscow, Russia) with heating $\left(70^{\circ} \mathrm{C}\right)$ for $30 \mathrm{~min}$. Then the samples were centrifuged for $5 \mathrm{~min} .5 \mathrm{ml}$ of the upper layer were collected in a test tube and centrifuged over again for $5 \mathrm{~min}$. Then, $2 \mathrm{ml}$ of the upper layer were collected with a medical syringe and filtered through Chromafil AO 45/3 filter (MACHEREY-NAGEL GmbH \& Co. KG, Düren, Germany). The resulting sample was twofold diluted by the water and then subjected to electrophoretic analysis. Each sample was analyzed three times and averaged profiles were further taken into account.

\subsection{Potentiometric multisensor system}

The multisensor system consisted of 17 chemical sensors. All sensors: 5 anion-sensitive, 7 cation-sensitive with polymeric membranes and 4 chalcogenide glass sensors with RedOx sensitivity were obtained from Sensor Systems, LLC (St. Petersburg, Russia). The system was also equipped with standard pH glass electrode and $\mathrm{Ag} / \mathrm{AgCl}$ reference electrode, both from ZIP (Gomel, Belorussia). All sensors were connected by shielded cables to the 32-channel high input impedance digital mV-meter (Sensor Systems, LLC, St. Petersburg, Russia). Potential readings were measured with $0.1 \mathrm{mV}$ precision and recorded by custom made software installed on a PC.

Original herbal brews evaluated by tasters were twenty-fold diluted for potentiometric measurements. The measurement time in TCM samples was 3 minutes. After that the sensors were washed by one portion of $0.05 \%$ TWEEN 20 for 1 min and two portions of distilled water for 2 min each. All samples were measured at least four times each and these results were averaged over the replicas for further processing. The resultant data matrix from the multisensor system comprised 33 samples $\times 17$ sensors.

\subsection{Data processing}

PLS algorithm [32] was used to construct calibration models from multisensor system measurements for quantitative assessment of bitterness and total content of polyphenols and caffeine in TCM samples. Reference data on bitterness intensity was obtained from the professional sensory panel whilst the content of polyphenols and caffeine was obtained using capillary electrophoresis.

PLS models were computed using The Unscrambler ${ }^{\circledR} 9.7$ (CAMO Software AS, Norway).

In order to confirm the validity of PLS regression models we employed cross-validation, independent test sets [32,34] and permutation testing [33]. In case of HPLC and CE data processing variable selection [35] was performed on the basis of regression coefficients of the variables in the models. Root mean squared error of cross-validation (RMSECV) and root mean squared error of prediction (RMSEP) were employed as metrics for predictive performance of the models. All three data sets (chromatographic, electrophoretic and potentiometric) were mean-centered before PLS modeling.

\section{Results and discussion}

\subsection{HPLC analysis}

Three different versions of liquid-liquid extraction-organic, water-organic and aqueous were employed to extract the components of TCM.

During organic extraction $100 \mathrm{mg}$ of crushed plant and $1 \mathrm{ml}$ of acetonitrile were vortex mixed for $15 \mathrm{~min}$. Then the samples were centrifuged for $10 \mathrm{~min}$ and $600 \mu \mathrm{l}$ the upper layer and $400 \mu \mathrm{l}$ of water were transferred into glass auto-sampler vials. These solutions were subjected to chromatographic analysis.

The samples consisting of $100 \mathrm{mg}$ of crushed plant and $2.5 \mathrm{ml}$ of $80 \%$ methanol were used for water-organic extraction. The samples were placed in ultrasonic bath with heating $\left(+50^{\circ} \mathrm{C}\right)$ for $30 \mathrm{~min}$. Then each sample was thoroughly mixed by vortex for $10 \mathrm{~min}$, centrifuged for $10 \mathrm{~min}$ and $2 \mathrm{ml}$ of upper layer were collected in a vial for evaporation to dryness under nitrogen stream. The dry residue was dissolved in $500 \mu$ l of $60 \%$ acetonitrile and then was subjected to chromatographic analysis.

The aqueous extraction was performed using $0.2 \%$ acetic acid. Acidic medium is necessary for transformation of charged analytes into their corresponding uncharged forms to provide for retention by chromatographic column [36]. A sample comprising $100 \mathrm{mg}$ of a plant and $1.5 \mathrm{ml}$ of $0.2 \%$ acetic acid was placed in ultrasonic bath with heating for $30 \mathrm{~min}$. Then the sample was thoroughly mixed by vortex for $10 \mathrm{~min}$ and centrifuged for $10 \mathrm{~min}, 1 \mathrm{ml}$ of the upper layer being further collected and diluted with $2 \mathrm{ml}$ of ethyl acetate. This mixture was vortex mixed for $10 \mathrm{~min}$ and another $1.6 \mathrm{ml}$ of the upper layer was collected in a vial and evaporated to dryness under a nitrogen stream. The dry residue was dissolved in $500 \mu \mathrm{l}$ of $60 \%$ acetonitrile and then was subjected to chromatographic analysis.

There are literature data [37] mentioning that TCM preparations may contain anthraquinones. To verify this fact, all samples were analyzed under chromatographic conditions proposed in 
a)

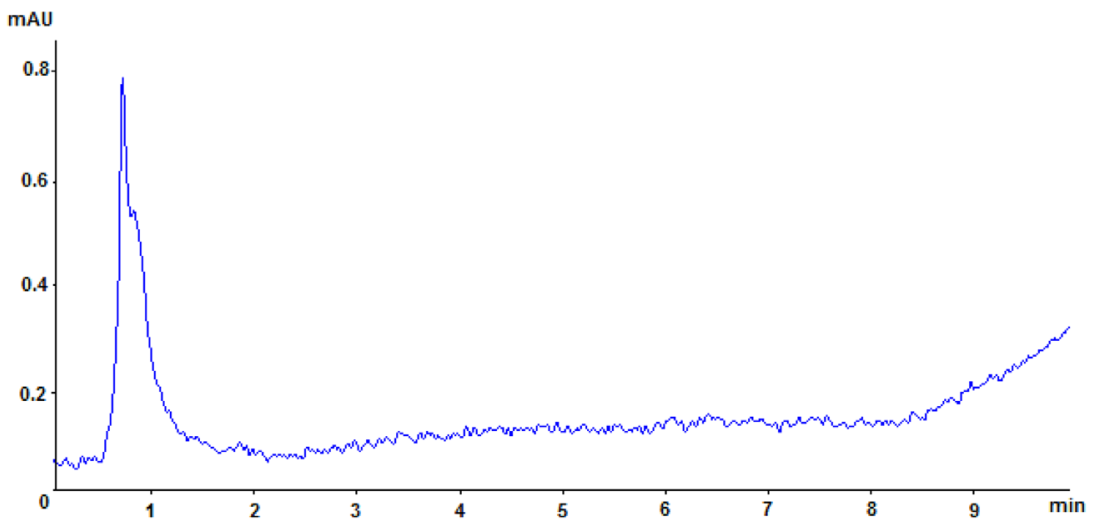

b)

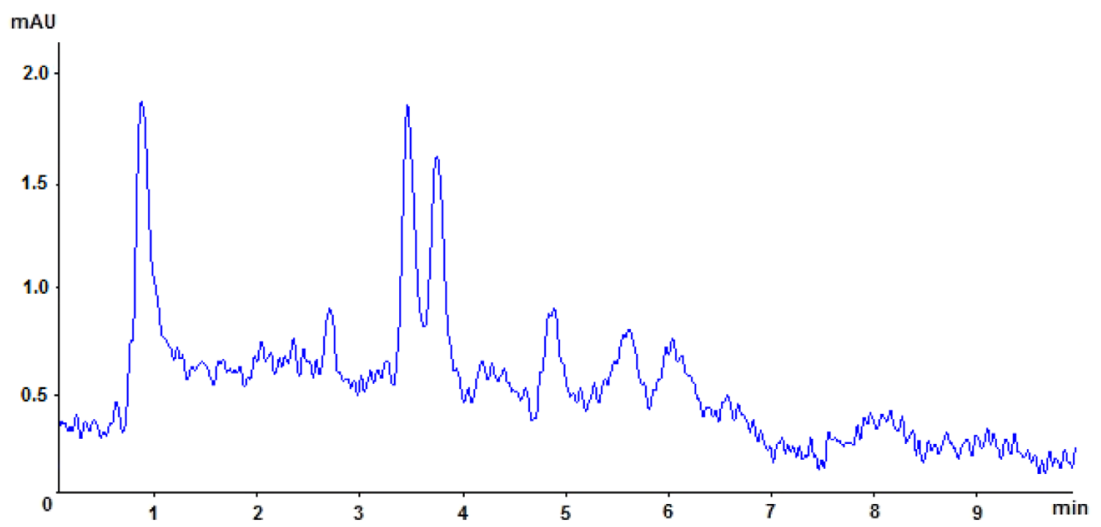

c)

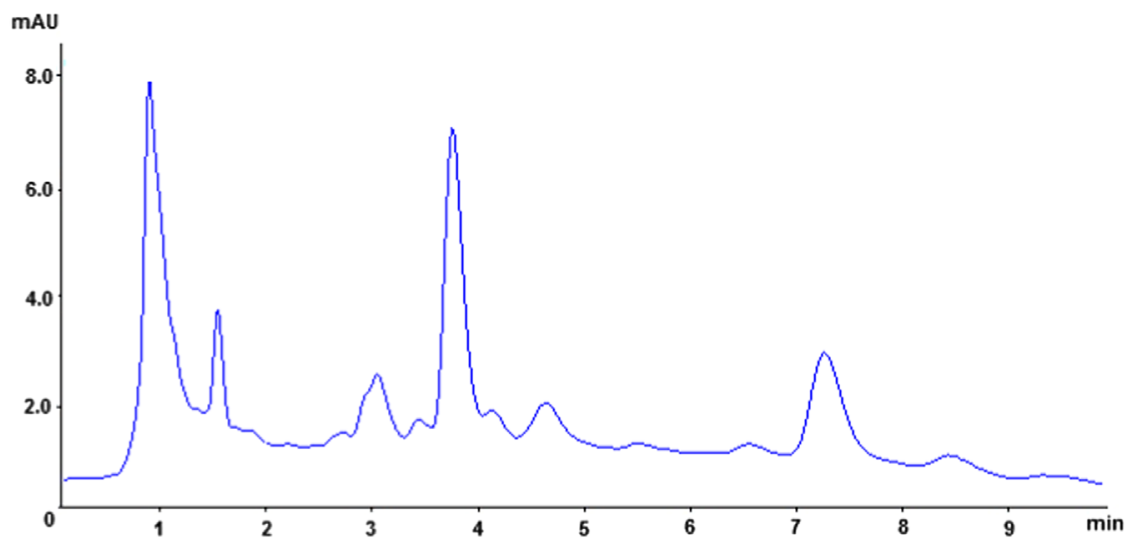

Fig. 1. Chromatogramms of Rehmanniae Adhesive sample: (a) aqueous, (b) water-organic and (c) organic extraction. UV detection at $254 \mathrm{~nm}$. Conditions: «Agilent-1200", YMC-Triart C18 column $(250 \times 4.6 \mathrm{~mm}, 5 \mu \mathrm{m})$; mobile phase $-60 \%$ acetonitrile and $0.05 \mathrm{M}$ formic acid in water; flow rate $-0.2 \mathrm{ml} / \mathrm{min}$; sample injection $-40 \mu \mathrm{l}$.

[36]. However, the targeted analytes (rhein and 1,8-dihydroxyanthraquinone) in the studied samples were not detected employing any sample preparation methods.

Fig. 1 shows typical chromatographic profiles obtained for the samples prepared by three different extraction modes. The acetonitrile extraction was chosen for further experiments since in this case the number of registered peaks was the highest. Corresponding chromatographic profiles were obtained for all TCM samples. The time to acquire chromatograms in such a case was 20 min rather than 10 min used in preliminary experiments.

These profiles were treated with PLS regression to relate them to bitter taste intensity assessed by human sensory panel. A similar idea was tested in [38], however the authors of [38] have identified and quantified individual compounds from chromatograms and they did not try to predict sensory descriptors numerically. Unlike conventional chromatographic approach we did not assign the peaks and did not analyze the content of particular substances in the samples. Instead we employed the whole chromatographic profile ("unresolved" raw data) as input for PLS modeling. In order to improve the quality of the regression models, variable selection was performed on the basis of the values of regression coefficients of corresponding variables [35] using RMSECV as a performance metric. During this procedure irrelevant and noisy variables were removed from the processing. This led to 
substantial decrease in the number of variables; only 1290 out of initial 5150 were kept. These were the segments of initial chromatographic profile corresponding to retention times in the intervals 3-6 min, 13-14 and 16-17 min. While further tests with polyphenolic standards (epicatechin, gallic acid, epigallocatechin, epigallocatechin gallate) confirmed that the segment of 3-6 min is due to these substances, the nature of two other segments and their impact on bitterness remained unclear. The parameters of the optimized PLS models (RMSECV $=0.67$ for bitterness range $0-6$ and $R^{2}=0.87$ ) allow suggesting that this instrumental chromatographic approach can be employed for bitter taste prediction in TCM. Since the number of samples was quite limited and crossvalidation may potentially yield overoptimistic results we also validated the models with permutation testing [33]. Fig. 1S (Supplementary material) shows the RMSECV value evolution through 50 permutations in Y-column (bitterness score from sensory panel). This result confirms the validity of the models.

\subsection{CE analysis}

The substances responsible for the bitter taste in plant materials are generally polyphenolic compounds and alkaloids (mainly caffeine) [39]. Optimal conditions for electrophoretic detection of polyphenols and caffeine in tea samples were suggested in [40]. Taking into account obvious similarity between tea and TCM samples we employed the methodology from [40] in our research. Fig. 2 shows electropherogram for the mixed standard solution of polyphenolics and caffeine.

TCM samples contain about ten times less polyphenols and caffeine compared to the content of these in tea samples. This implies the relevance of extraction procedure described above in Section 2.5. All TCM samples were analyzed by CE and corresponding electrophoretic profiles were obtained. Fig. 3 shows the electrophoretic profile of a Chinese Coptis sample, as an example.

CE was employed for quantitative assessment of polyphenols and caffeine in all samples after performing calibration of UV detector using a series of standard polyphenolic and caffeine solutions. The detection limits under experimental conditions were calculated as triple noise signal and were $1 \mathrm{mg} / \mathrm{L}$ for all polyphenols and caffeine. Quantification limits were $10 \mathrm{mg} / \mathrm{L}$ for Caf, EGC, EGCG and $5 \mathrm{mg} / \mathrm{L}$ for EC and GA. The results of quantitative assessment of polyphenols and caffeine in the samples are shown in Table 2.

The whole electrophoretic profiles were treated with PLS regression to relate them to bitter taste intensity scores from human sensory panel just like in the case with HPLC. Variable selection was also performed based on regression coefficients in cross- validated PLS models. 670 variables describing time segments corresponding to the polyphenols and caffeine (out of 6800 in total) were kept for analysis. The parameters of the optimized PLS models (RMSECV $=0.85$ for bitterness range $0-6$ and $R^{2}=0.82$ ) were somewhat worse than those for HPLC, however still permitting numerical prediction of bitterness score. Permutation testing confirmed the validity of the models (Fig. 2S, Supplementary material).

\subsection{ET analysis}

In order to obtain reproducible values of sensor signals it was necessary to establish the optimal sample dilution and washing procedures. A number of experiments were performed to select the most appropriate dilution rate. The idea behind this experiment is that too much concentrated media can cause excessive adsorption of the constituents on the membrane surface and subsequent deterioration of sensor response. To study this issue the sample concentration in the water was varied over three levels: $5 \%, 10 \%$ and $20 \%$. A mixture of four plants (sample 33 , Table 1 ) was employed as a trial sample. Appropriate portions of the sample were measured five times at each dilution rate by multisensor system and sensor readings in these replicated measurements were registered. Sensors responses in the final washing water between the measurements were also recorded. The stability of sensor potentials in the water is a good indicator of the sensor system stability.

The standard deviations over five measurements in TCM sample and those of the five corresponding water wash samples were calculated for each sensor. Table 2S (Supplementary material) demonstrates the sums of standard deviation values for all of the sensors for three different sample concentrations.

The minimal sum of standard deviations was obtained for the lowest content of the sample - 5\%. This dilution rate was therefore used in all subsequent experiments.

It was found out during the other trial measurements that washing of the sensor array by $0.05 \%$ TWEEN-20 ensures better reproducibility of the replicated measurements. Thus, the resulted washing procedure included $1 \mathrm{~min}$ of washing by TWEEN and two cycles of washing by distilled water for 2 min each. TWEEN is a typical non-ionogenic surface active substance and it likely helps to wash away highly adhesive components of a sample from the sensor membrane surface thus promoting better reproducibility.

Since polyphenols and alkaloids are the main substances responsible for bitter taste in plants brews, we tried to relate the response of multisensor system to the sum of polyphenols and caffeine concentrations in the studied samples. This was done by

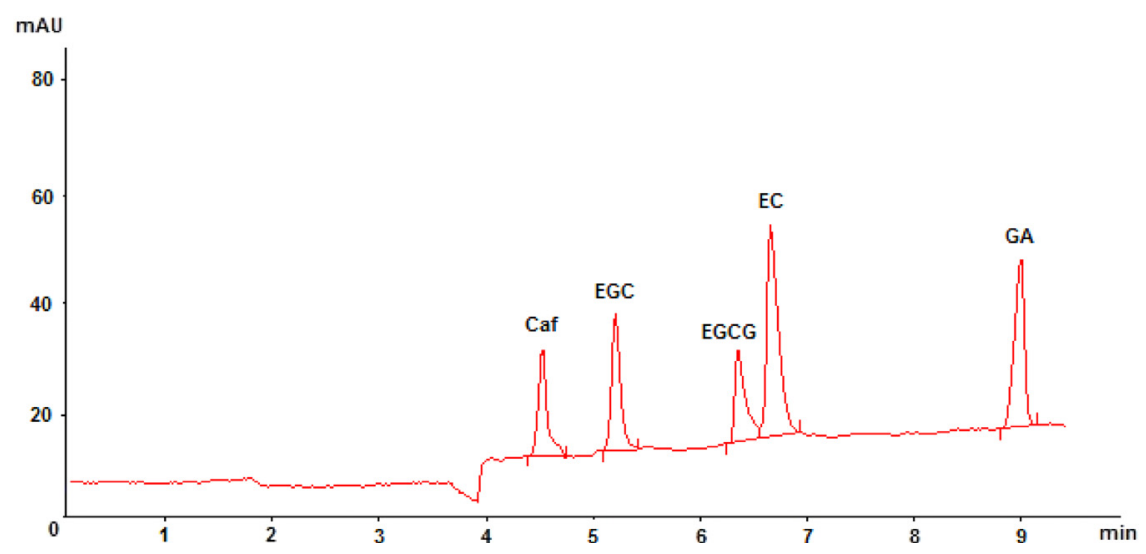

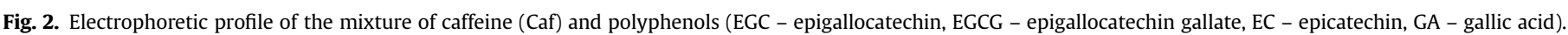

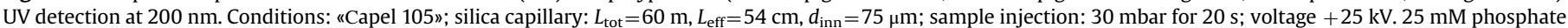
buffer $(\mathrm{pH}=7.0)$ and $25 \mathrm{mM}$ sodium dodecyl sulfate. 


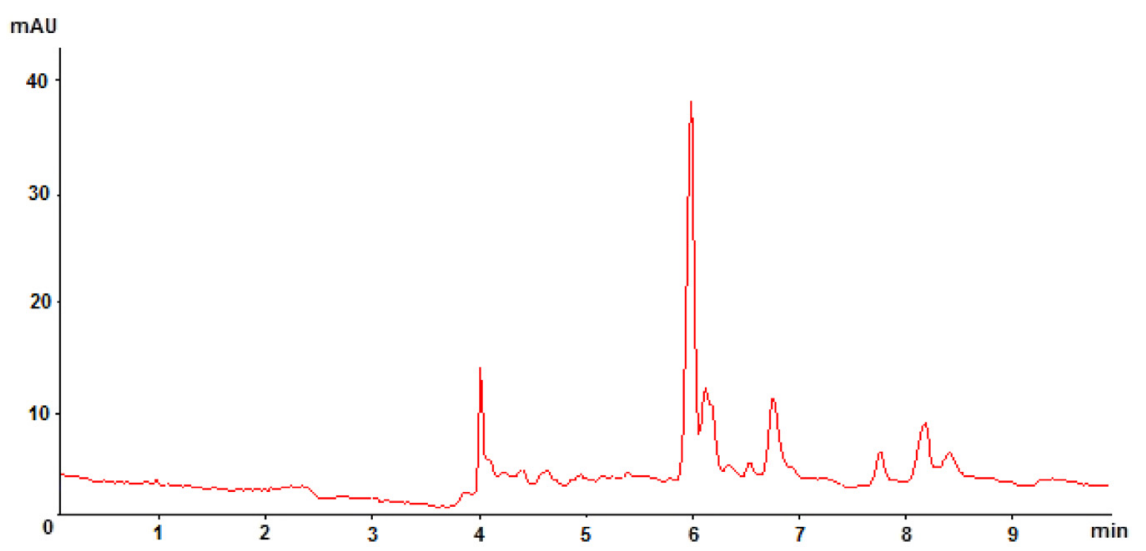

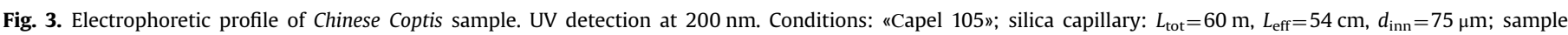
injection: 30 mbar for $20 \mathrm{~s}$; voltage $+25 \mathrm{kV}$; analysis time $-10.5 \mathrm{~min}$. $25 \mathrm{mM}$ phosphate buffer (pH=7.0) and $25 \mathrm{mM}$ sodium dodecyl sulfate.

the PLS regression where CE data on these substances were employed as reference data. Validation was performed using test set of 9 randomly chosen samples. Table 3 summarizes the parameters of the model. It was found that potentiometric multisensor system can determine the sum of polyphenols and caffeine in TCM with RMSEP 0.09 wt\%.

In the case of the multisensor system it was possible to use the same samples that were analyzed by sensory panel due to simple sample preparation procedures (Table 1), thus the total number of samples amounted to 33 . The data from the multisensor system were employed for PLS modeling in order to predict bitter taste intensity. 23 samples were used as a calibration set and 10 randomly chosen samples were included in the independent test set to evaluate the predictive performance of the model. The resulted "measured vs. predicted plot" is given in the Fig. 4 and model parameters are shown in Table 3.

Reasonable precision of bitter taste prediction from multisensor system response can be attained. The RMSEP value (0.86) for e-tongue is comparable to those of $C E(R M S E C V=0.85)$ and HPLC (RMSECV $=0.67$ ). Taking into account the very simple sample preparation for multisensor system and overall simplicity and rapidity of the measurement method it could be stated that this is a preferable option for instrumental bitter taste assessment in TCM. It is noteworthy that we compared here RMSEP and RMSECV values since only a lower number of samples was available for HPLC and $\mathrm{CE}$ due to features of sample preparation.

\section{Conclusions}

Three different analytical instruments were applied for bitterness evaluation of TCM herbs - HPLC coupled to UV detector, CE coupled to UV detector and potentiometric multisensor system electronic tongue. Sample preparation conditions were optimized
Table 3

Parameters of the measured vs. predicted plot for PLS-regression models for multisensor system data.

\begin{tabular}{|c|c|c|c|c|}
\hline & Slope & Offset & RMSE & $R^{2}$ \\
\hline \multicolumn{5}{|c|}{ Total content of polyphenols and caffeine (0.02-1.00 wt\%) } \\
\hline Calibration & 0.96 & 0.01 & 0.07 & 0.97 \\
\hline Validation & 0.86 & 0.02 & 0.09 & 0.87 \\
\hline \multicolumn{5}{|c|}{ Bitterness (0-6 points) } \\
\hline Calibration & 0.84 & 0.38 & 0.62 & 0.92 \\
\hline Validation & 0.86 & 0.37 & 0.86 & 0.83 \\
\hline
\end{tabular}

to obtain information-rich chromatographic profiles. Derived chromatographic and electrophoretic profiles as well as ET data were processed employing PLS regression and it was shown that all three methods are capable of quantitative assessment of bitter taste in TCM samples with reasonable precision. HPLC and CE require quite long and tedious sample preparation even if employed in "profile" mode without peak assignment, while potentiometric ET requires very simple sample pretreatment, basically some dilution, and attains the same precision of bitterness score prediction as HPLC and CE. Nevertheless, application of HPLC in CE in "unresolved profile" mode in combination with chemometric data processing can obviously be very helpful in instrumental taste assessment for other applications where taste stimuli are generated due to diverse chemical compounds.

\section{Acknowledgment}

This work was partially financially supported by Government of Russian Federation (Grant 074-U01) and Russian Foundation for Basic research (RFBR) 15-53-53051 GFEN_a.

Table 2

The contents of polyphenols and caffeine in TCM samples according to CE analysis, wt $\%(P=0.95, n=3)$. n/d - not detectable.

\begin{tabular}{|c|c|c|c|c|c|}
\hline Sample & Caf & EGC & EGCG & EC & GA \\
\hline Chinese Chrysanthemum & $\mathrm{n} / \mathrm{d}$ & $0.327 \pm 0.014$ & $0.526 \pm 0.009$ & $0.156 \pm 0.009$ & $\mathrm{n} / \mathrm{d}$ \\
\hline Liquorice Root & $0.073 \pm 0.004$ & 0.000 & $0.098 \pm 0.003$ & $0.257 \pm 0.008$ & $\mathrm{n} / \mathrm{d}$ \\
\hline Cassia Twig & $\mathrm{n} / \mathrm{d}$ & $0.046 \pm 0.005$ & $\mathrm{n} / \mathrm{d}$ & $0.045 \pm 0.002$ & $0.300 \pm 0.005$ \\
\hline Chinese Ephedra & $0.199 \pm 0.009$ & $\mathrm{n} / \mathrm{d}$ & $\mathrm{n} / \mathrm{d}$ & $\mathrm{n} / \mathrm{d}$ & $\mathrm{n} / \mathrm{d}$ \\
\hline Bitter Apricot Seeds & $0.108 \pm 0.008$ & $0.334 \pm 0.009$ & $\mathrm{n} / \mathrm{d}$ & $\mathrm{n} / \mathrm{d}$ & $\mathrm{n} / \mathrm{d}$ \\
\hline Rehmanniae Adhesive & $0.044 \pm 0.004$ & $0.016 \pm 0.002$ & $0.034 \pm 0.001$ & $\mathrm{n} / \mathrm{d}$ & $\mathrm{n} / \mathrm{d}$ \\
\hline Dandelion Root & $0.022 \pm 0.001$ & $\mathrm{n} / \mathrm{d}$ & $0.058 \pm 0.002$ & $\mathrm{n} / \mathrm{d}$ & $0.221 \pm 0.007$ \\
\hline Chinese Coptis & $\mathrm{n} / \mathrm{d}$ & $0.466 \pm 0.011$ & $\mathrm{n} / \mathrm{d}$ & $0.019 \pm 0.001$ & $\mathrm{n} / \mathrm{d}$ \\
\hline
\end{tabular}




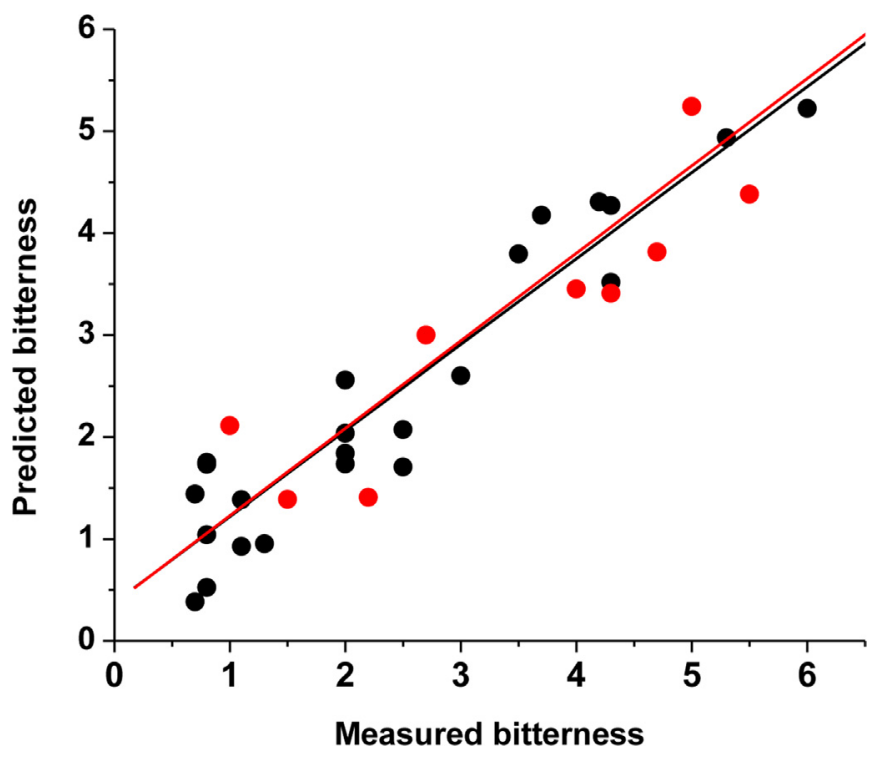

Fig. 4. Calibration (black points) and validation (red points) of the PLS model for prediction of bitter taste intensity in TCM samples from multisensor system response. (For interpretation of the references to color in this figure legend, the reader is referred to the web version of this article)

\section{Appendix A. Supplementary material}

Supplementary data associated with this article can be found in the online version at http://dx.doi.org/10.1016/j.talanta.2016.01. 058.

\section{References}

[1] L. Li, Biologically active components from traditional Chinese medicines, Pure Appl. Chem. 70 (3) (1998) 547-554.

[2] Y. Jiang, B. David, P. Tu, Y. Barbin, Recent analytical approaches in quality control of traditional Chinese medicines-a review, Anal. Chim. Acta 657 (2010) $9-18$.

[3] M. Li, X. Hou, J. Zhang, S. Wang, Q. Fu, L. He, Applications of HPLC/MS in the analysis of traditional Chinese medicines, J. Pharm. Anal. 1 (2011) 81-91.

[4] M. Kataoka, E. Tokuyama, Y. Miyanaga, T. Uchida, The taste sensory evaluation of medicinal plants and Chinese medicines, Int. J. Pharm. 351 (2008) 36-44.

[5] R. Liu, X. Zhang, L. Zhang, X. Gao, H. Li, J. Shi, X. Li, Bitterness intensity prediction of berberine hydrochloride using an electronic tongue and a GA-BP neural network, Exp. Ther. Med. 7 (2014) 1696-1702.

[6] Y. Wang, Y. Feng, Y. Wu, S. Liang, D. Xu, Sensory evaluation of the taste of berberine hydrochloride using an Electronic Tongue, Fitoterapia 86 (2013) 137-143.

[7] A. Gonzalo-Diago, M. Dizy, P. Fernandez-Zurbano, Contribution of low molecular weight phenols to bitter taste and mouthfeel properties in red wines, Food Chem. 154 (2014) 187-198.

[8] R. Ferrer-Gallego, J. Hernández-Hierro, J. Rivas-Gonzalo, M. Escribano-Bailón, Sensory evaluation of bitterness and astringency sub-qualities of wine phenolic compounds: synergistic effect and modulation by aromas, Food Res. Int. 62 (2014) 1100-1107.

[9] S. Kallithraka, D. Kim, A. Tsakiris, I. Paraskevopoulos, G. Soleas, Sensory assessment and chemical measurement of astringency of Greek wines: correlations with analytical polyphenolic composition, Food Chem. 126 (2011) 1953-1958.

[10] Q. Chen, J. Zhao, Z. Guo, X. Wang, Determination of caffeine content and main catechins contents in green tea (Camellia sinensis L.) using taste sensor technique and multivariate calibration, J. Food Compos. Anal. 23 (2010) 353-358.

[11] H. Horie, T. Mukai, K. Kohata, Simultaneous determination of qualitatively important components in green tea infusions using capillary electrophoresis, J. Chromatogr. A 758 (1997) 332-335.

[12] D. Horzic, D. Komes, A. Belšcak, K. Ganic, D. Ivekovic, D. Karlovic, The composition of polyphenols and methylxanthines in teas and herbal infusions,
Food Chem. 115 (2009) 441-448.

[13] J. Hu, Y. Chen, D. Ni, Effect of superfine grinding on quality and antioxidant property of fine green tea powders, LWT - Food Sci. Technol. 45 (2012) 8-12.

[14] L. Kerio, F. Wachira, J. Wanyoko, M. Rotich, Total polyphenols, catechin profiles and antioxidant activity of tea products from purple leaf coloured tea cultivars, Food Chem. 136 (2013) 1405-1413.

[15] I. Hecimovic, A. Belšcak-Cvitanovic, D. Horžić, D. Komes, Comparative study of polyphenols and caffeine in different coffee varieties affected by the degree of roasting, Food Chem. 129 (2011) 991-1000.

[16] J. Ribeiro, M. Ferreira, T. Salva, Chemometric models for the quantitative descriptive sensory analysis of Arabica coffee beverages using near infrared spectroscopy, Talanta 83 (2011) 1352-1358.

[17] F. Favati, N. Condelli, F. Galgano, M. Caruso, Extra virgin olive oil bitterness evaluation by sensory and chemical analyses, Food Chem. 139 (2013) 949-954.

[18] A. Pripp, J. Busch, R. Vreeker, Effect of viscosity, sodium caseinate and oil on bitterness preception of olive oil phenolics, Food Qual. Prefer. 15 (2004) 375-382.

[19] L. Bartoshuk, Chem. Senses 25 (2000) 447-460.

[20] A. Rudnitskaya, E. Polshin, D. Kirsanov, J. Lammertyn, B. Nicolai, D. Saison, F. Delvaux, F. Delvaux, A. Legin, Instrumental measurement of beer taste attributes using an electronic tongue, Anal. Chim. Acta 646 (2009) 111-118.

[21] H. Smyth, D. Cozzolino, Instrumental methods (spectroscopy, electronic nose, and tongue) as tools to predict taste and aroma in beverages: advantages and limitations, Chem. Rev. 113 (3) (2013) 1429-1440.

[22] J. Newman, T. Egan, N. Harbourne, D. O’Riordan, J.C. Jacquier, M. O’Sullivan, Correlation of sensory bitterness in dairy protein hydrolysates: comparison of prediction models built using sensory, chromatographic and electronic tongue data, Talanta 126 (2014) 46-53.

[23] M. Saenz-Navajas, E. Campo, L. Cullere, P. Fernandez-Zurbano, D. Valentin, $\mathrm{V}$. Ferreira, Effects of the nonvolatile matrix on the aroma perception of wine, J. Agric. Food Chem. 58 (2010) 5574-5585.

[24] D. Cozzolino, H. Smyth, K. Lattey, W. Cynkar, L. Janik, R. Dambergs, L. Francis, M. Gishen, Combining mass spectrometry based electronic nose, visible-near infrared spectroscopy and chemometrics to assess the sensory properties of Australian Riesling wines, Anal. Chim. Acta 563 (2006) 319-324.

[25] I. Esteban-Diez, J. Gonzalez-Saiz, C. Pizarro, Prediction of sensory properties of espresso from roasted coffee samples by near-infrared spectroscopy, Anal. Chim. Acta 525 (2004) 171-182.

[26] R. Karoui, L. Pillonel, E. Schaller, J. Bosset, J. Baerdemaeker, Prediction of sensory attributes of European Emmental cheese using near-infrared spectroscopy: a feasibility study, Food Chem. 101 (2007) 1121-1129.

[27] Q. Wang, S. Lonergan, C. Yu, Rapid determination of pork sensory quality using Raman spectroscopy, Meat Sci. 91 (2012) 232-239.

[28] J. Broadbent, M. Strickland, B. Weimer, M. Johnson, J. Steele, Peptide Accumulation and bitterness in cheddar cheese made using single-strain lactococcus lactis starters with distinct proteinase specificities, J. Dairy Sci. 81 (2) (1998) 327-337.

[29] L. Lvova, D. Kirsanov, A. Legin, C. Di Natale, Multisensor Systems for Chemical Analysis - Materials and Sensors, Pan Stanford Publishing, Singapore, 2014.

[30] J. Zheng, M. Keeney, Taste masking analysis in pharmaceutical formulation development using an electronic tongue, Int. J. Pharm. 310 (2006) 118-124.

[31] A. Rudnitskaya, D. Kirsanov, Y. Blinova, E. Legin, B. Seleznev, D. Clapham, R. Ives, K. Saunders, A. Legin, Assessment of bitter taste of pharmaceuticals with multisensor system employing 3 way PLS regression, Anal. Chim. Acta 770 (2013) 45-52.

[32] K. Esbensen, Multivariate Data Analysis - in Practice, CAMO Software, Oslo, 2002.

[33] B. LaFleur, R. Greevy, Introduction to permutation and resampling-based hypothesis tests, J. Clin. Child Adolesc. 38 (2) (2009) 286-294.

[34] I. Scott, W. Lin, M. Liakata, J. Wood, C. Vermeer, D. Allaway, J. Ward, J. Draper, M. Beale, D. Corol, J. Baker, R. King, Merits of random forests emerge in evaluation of chemometric classifiers by external validation, Anal. Chim. Acta 801 (2013) 22-33.

[35] C Andersen, R. Bro, Variable selection in regression-a tutorial, J. Chemometr. 24 (2010) 728-737.

[36] I. Yaroshenko, A. Khaimenov, A. Grigoriev, A. Sidorova, Determination of Rhein in blood plasma by HPLC with UV detection and its application to the study of bioequivalence, J. Anal. Chem. 69 (8) (2014) 793-799.

[37] J. Jiang, M. Yang, W. Qian, H. Lin, Y. Geng, Z. Zhou, D. Xiao, Quantitative determination of rhein in human plasma by liquid chromatography-negative electrospray ionization tandem mass/mass spectrometry and the application in a pharmacokinetic study, J. Pharm. Biomed. Anal. 57 (2012) 19-25.

[38] I. Stanimirova, C. Bouconb, B. Walczak, Relating gas chromatographic profiles to sensory measurements describing the end products of the Maillard reaction, Talanta 83 (2011) 1239-1246.

[39] J. Phillipson, Phytochem. Pharmacogn. Phytochem. 68 (2007) 2960-2972.

[40] L. Kartsova, A. Alekseeva, Chromatographic and electrophoretic methods for determining polyphenol compounds, J. Anal. Chem. 63 (2008) 1024-1033. 